\title{
Paclitaxel-Coated Zilver PTX Drug-Eluting Stent Treatment Does Not Result in Increased Long-Term All-Cause Mortality Compared to Uncoated Devices
}

\author{
Michael D. Dake ${ }^{1}$ - Gary M. Ansel ${ }^{2} \cdot$ Marc Bosiers $^{3} \cdot$ Andrew Holden $^{4}$. \\ Osamu Iida ${ }^{5}$ Michael R. Jaff ${ }^{6} \cdot$ Aaron E. Lottes $^{7} \cdot$ Erin E. O'Leary ${ }^{7}$ • \\ Alan T. Saunders ${ }^{7} \cdot$ Marc Schermerhorn $^{8} \cdot$ Hiroyoshi Yokoi $^{9} \cdot$ Thomas Zeller $^{10}$
}

Received: 24 July 2019/Accepted: 21 August 2019/Published online: 9 September 2019

(C) The Author(s) 2019

\begin{abstract}
Purpose Patient-level data from two large studies of the Zilver PTX drug-eluting stent (DES) with long-term follow-up and concurrent non-drug comparator groups were analyzed to determine whether there was an increased mortality risk due to paclitaxel.

Methods Data from the Zilver PTX randomized controlled trial (RCT) and Zilver PTX and bare metal stent (BMS) Japan post-market surveillance studies were analyzed.
\end{abstract}

Electronic supplementary material The online version of this article (https://doi.org/10.1007/s00270-019-02324-4) contains supplementary material, which is available to authorized users.

Michael D. Dake

mddake@email.arizona.edu

1 The University of Arizona, Roy P. Drachman Hall Building, B207, 1295 North Martin Avenue, P.O. Box 210202, Tucson, AZ 85721-0202, USA

2 Department of Medicine, Ohio Health/Riverside Methodist Hospital, Columbus, OH, USA

3 Foundation of Cardiovascular Research and Education, Münster, Germany

4 Department of Interventional Radiology, Auckland City Hospital, Auckland, New Zealand

5 Cardiovascular Center, Kansai Rosai Hospital, Amagasaki, Japan

6 Harvard Medical School, Boston, MA, USA

7 Cook Research Incorporated, West Lafayette, IN, USA

8 Division of Vascular Surgery, Department of Surgery, Beth Israel Deaconess Medical Center, Boston, MA, USA

9 Department of Cardiovascular Medicine, Fukuoka Sanno Hospital, Fukuoka, Japan

10 Universitaets-Herz-Zentrum Freiburg - Bad Krozingen, Bad Krozingen, Germany
Five-year follow-up is complete in both DES studies; follow-up for the BMS study was limited to 3 years and is complete. Kaplan-Meier analyses assessed mortality. A Cox proportional hazards model identified significant factors related to mortality.

Results In the RCT, there were 336 patients treated with the DES and 143 patients treated with percutaneous transluminal angioplasty (PTA) or BMS. In Japan, there were 904 DES patients and 190 BMS patients. There was no difference in all-cause mortality for the DES compared to PTA/BMS in the RCT (19.1\% DES versus $17.1 \%$ PTA/ BMS through 5 years, $p=0.60)$ or Japan $(15.8 \%$ DES versus $15.3 \%$ BMS through 3 years, $p=0.89$ ). Cox proportional hazard models revealed that age, tissue loss, and congestive heart failure were significantly associated with mortality in the RCT, and critical limb ischemia, age, renal failure, and gender were significantly associated with mortality in Japan (all $p<0.05$ ). Neither treatment with Zilver PTX ( $p=0.46$ RCT, $p=0.49$ Japan) nor paclitaxel dose ( $p=0.86 \mathrm{RCT}, p=0.07$ Japan) was associated with mortality.

Conclusion Analyses of the Zilver PTX patient-level data demonstrated no increase in long-term all-cause mortality. Level of Evidence Zilver PTX RCT: Level 1, randomized controlled trial; Japan PMS studies: Level 3, post-market surveillance study.

Keywords Paclitaxel · Drug-eluting stent - Bare metal stent - Percutaneous transluminal angioplasty . Mortality $\cdot$ Peripheral artery disease 


\section{Introduction}

An endovascular-first approach is commonly used to treat symptomatic femoropopliteal peripheral artery disease (PAD) $[1,2]$. Over nearly a decade, drug-device combination technologies using the antiproliferative agent, paclitaxel, have been developed to reduce restenosis rates compared to percutaneous transluminal angioplasty (PTA) or bare metal stents (BMS) [3-11].

Recently, a meta-analysis that grouped both drug-eluting stents (DES) and drug-coated balloons (DCB) together indicated a higher incidence of late all-cause mortality for paclitaxel-based devices compared to uncoated PTA or BMS at 2 years up to 5 years [12]. This difference in mortality was attributed directly to paclitaxel although no etiology was postulated for the increased rate. The authors reported a dose-dependent relationship in the mortality rate that was based on the dose density of each device rather than the actual amount of paclitaxel coated on the device.

The authors of the meta-analysis used data available in publications or other public online resources. For the Zilver PTX DES, the published data were an intent-to-treat summary analysis, which accounted for patients' treatment at the time of enrollment. However, due to the unique trial design of the Zilver PTX randomized controlled trial (RCT), patients were not limited to stent placement only at enrollment. To evaluate the long-term mortality rate of the DES, the patient-level data from the Zilver PTX RCT were analyzed based on treatment actually received, and the results for patients treated with the DES were compared to patients treated only with PTA and/or BMS. Supporting evidence from the Japan Zilver PTX and Zilver BMS postmarket studies (PMS) provides long-term mortality information from a broader patient population.

\section{Methods}

\section{Randomized Controlled Trial}

The Zilver PTX RCT was a prospective, multinational, randomized study comparing the safety and effectiveness of the polymer-free, paclitaxel-coated Zilver PTX DES (Cook Medical, Bloomington, IN, USA) to PTA and provisional BMS placement in patients with femoropopliteal PAD. Study characteristics have been previously described [3] and are summarized in Online Resource 1. Five-year follow-up is complete, and results have been published [3-5]. Patients with symptomatic PAD involving the above-the-knee femoropopliteal arteries were initially randomized to PTA or to stent placement with the paclitaxel-coated Zilver PTX DES. Patients with acute PTA failure (e.g., $\geq 30 \%$ residual stenosis remaining after minimum of additional 2- to 3-min balloon inflation) underwent a secondary randomization and were treated with either the DES or a BMS (Zilver, Cook Medical, Bloomington, IN, USA). PTA patients and BMS patients who required re-intervention within the first year postprocedure were permitted to cross over to treatment with the DES. Thus, there were three opportunities to receive a DES: two via protocol-specified randomization and the third via crossover due to subsequent failure of an initially successful treatment (note, patients had reached the study endpoint for efficacy). Recognizing missing data as a limitation, vital status for patients who did not originally complete the study was ascertained; all available vital status data are included in the current mortality analyses.

\section{Japan Post-market Surveillance Study}

Post-market data were collected from a prospective, multicenter registry of the Zilver PTX DES as well as concurrent prospectively enrolled patients treated with the Zilver BMS (Online Resource 1).

The Japan DES PMS had no exclusion criteria and enrolled consecutive patients with symptomatic PAD involving the above-the-knee femoropopliteal arteries. Safety and effectiveness of the DES were evaluated in realworld patients with complex femoropopliteal artery lesions through 5 years $[13,14]$. Follow-up in the study is complete.

The concurrent Japan BMS PMS also had no exclusion criteria and enrolled consecutive patients with symptomatic PAD involving the above-the-knee femoropopliteal arteries. Patients who were enrolled in the BMS study but who also had a DES placed $(n=18)$ were excluded from the current analysis. Follow-up in the BMS study was only required through 3 years and is complete.

\section{All-Cause Mortality and Adverse Events}

All deaths reported during active study follow-up were adjudicated by an independent clinical events committee to determine the cause of death and relatedness to the procedure or device.

\section{Statistical Analysis}

Analyses were performed using SAS 9.4 (SAS Institute, Cary, NC, USA). All comparisons of the DES group to the PTA/BMS group were made based on treatment actually received. Continuous variables were summarized with means and standard deviations, with $p$ values calculated using the standard $t$ test. Categorical variables were reported as counts and percentages, with $p$ values 
calculated using Fisher's exact test. Kaplan-Meier analyses were performed to assess mortality. In the RCT analyses, day 0 was set as the day of crossover to DES treatment. $p$ values for the Kaplan-Meier analyses were calculated using the log-rank test. Cox proportional hazard models for paclitaxel treatment (binary) and paclitaxel dose (continuous; amount of paclitaxel received by each patient) were performed using the relevant covariates expected to have a potential impact on mortality. The model incorporated all covariates simultaneously to ascertain whether there was a potential confounding factor that could influence mortality beyond the factor in question, i.e., DES versus PTA/BMS. The patient-level data used for the RCT analyses presented here is available on the following website: https://www. cookmedical.com/peripheral-intervention/paclitaxel/.

\section{Results}

\section{Randomized Controlled Trial}

Of the 479 patients enrolled in the RCT, 237 patients were included in the primary PTA group and 242 patients were included in the primary DES group (Fig. 1). However, 63 patients who were randomized to primary PTA treatment received the DES following acute PTA failure, and an additional 31 patients received the DES as treatment for failure of their original therapy within the first year. As a result, 94 patients who were initially randomized to the primary PTA group were actually treated with the DES ( $40 \%$ of the primary PTA group received a DES). In total,
336 patients were treated with a DES and 143 patients were treated only with PTA and/or BMS (Fig. 1). Baseline patient and lesion characteristics are summarized in Table 1. Five-year mortality data were available for $94 \%$ of patients. There were 61 deaths in the DES group and 23 deaths in the PTA/BMS group through 5 years; none were adjudicated as procedure- or device-related. There was no difference in the 5-year Kaplan-Meier estimates for allcause mortality for the DES group compared to the PTA/ BMS group (19.1\% vs. $17.1 \%$, log-rank $p=0.60$; Fig. 2). There were no significant differences between the DES and PTA/BMS groups in causes of death (Table 2) or adverse events (Online Resource 2).

The Cox proportional hazards model revealed that age $(p<0.001)$, tissue loss $(p=0.02)$, and congestive heart failure $(p=0.02)$ were significantly associated with mortality through 5 years (Fig. 3). Treatment with Zilver PTX $(p=0.46)$ was not associated with mortality. In the covariate analysis of paclitaxel dose, the significant factors were identical to the treatment analysis, and there was no association or trend of paclitaxel dose $(p=0.86)$ with mortality (Online Resource 3).

\section{Japan Post-market Surveillance Study}

The Japan DES PMS enrolled 904 DES patients, and the Japan BMS PMS enrolled 190 BMS patients. Baseline patient and lesion characteristics for these patient groups are summarized in Table 3.

In the DES group, there were 127 deaths through 3 years and 186 deaths through 5 years. In the BMS group, there

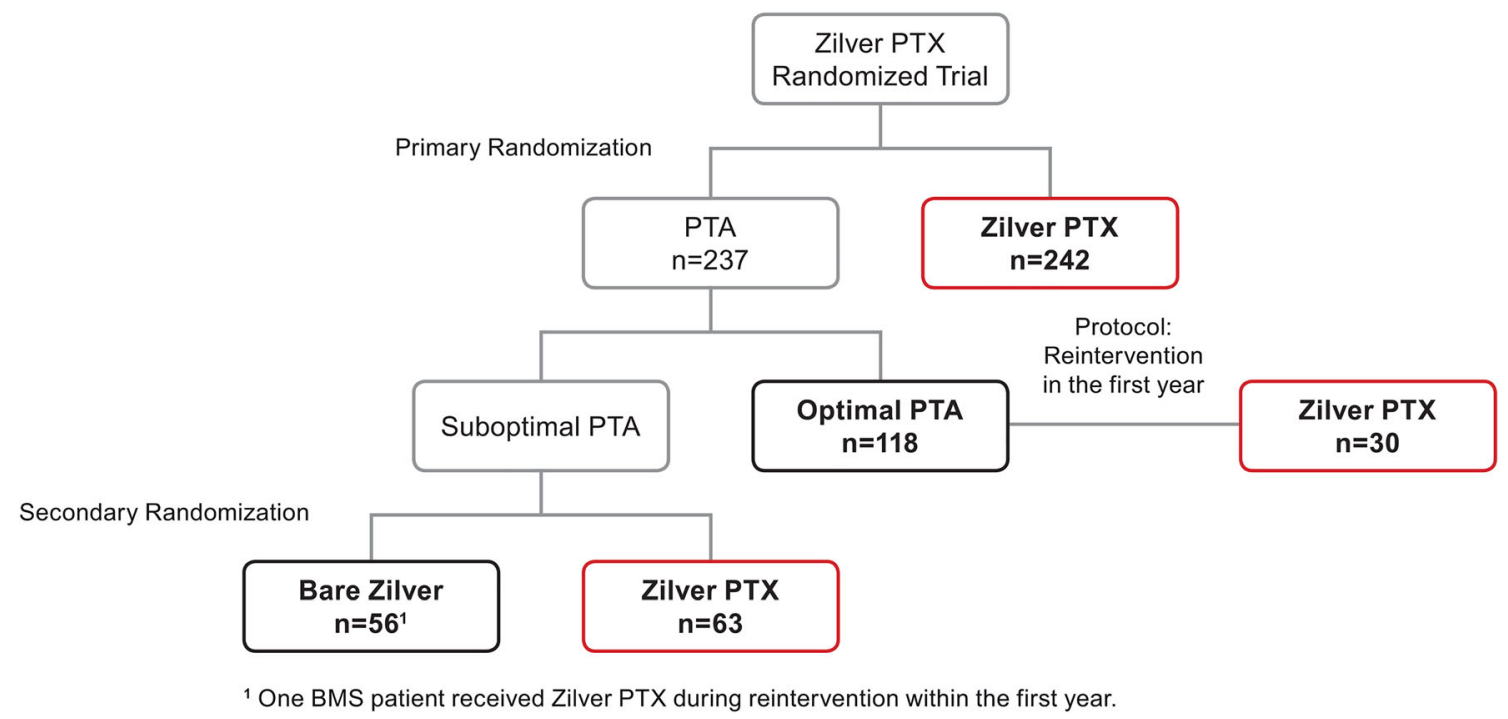

Fig. 1 Patient flow diagram for patients enrolled in the Zilver PTX RCT. Patient flow diagram based on actual treatment, with DES patients shown in red and PTA and BMS patients shown in black. Protocol specified that patients who experienced re-intervention within the first year could cross over to DES treatment. RCT, randomized controlled trial; DES, drug-eluting stent; PTA, percutaneous transluminal angioplasty; BMS, bare metal stent 
Table 1 Zilver PTX RCT demographics and baseline lesion characteristics

\begin{tabular}{|c|c|c|c|}
\hline Characteristic & DES & PTA/BMS & $p$ value \\
\hline Age (years) & $67.6 \pm 9.6(336)$ & $68.2 \pm 11.2(143)$ & 0.58 \\
\hline Gender & & & 0.60 \\
\hline Male & $65.8 \%(221 / 336)$ & $62.9 \%(90 / 143)$ & \\
\hline Female & $34.2 \%(115 / 336)$ & $37.1 \%(53 / 143)$ & \\
\hline Ethnicity & & & 0.96 \\
\hline Asian & $12.8 \%(38 / 298)$ & $14.0 \%(17 / 121)$ & \\
\hline Black/African-American & $12.1 \%(36 / 298)$ & $10.7 \%(13 / 121)$ & \\
\hline Hispanic/Latino & $6.7 \%(20 / 298)$ & $5.8 \%(7 / 121)$ & \\
\hline White/Caucasian & $68.5 \%(204 / 298)$ & $69.4 \%(84 / 121)$ & \\
\hline Body mass index & $28.3 \pm 5.2(336)$ & $28.4 \pm 6.0(143)$ & 0.87 \\
\hline Diabetes & $45.2 \%(152 / 336)$ & $46.9 \%(67 / 143)$ & 0.76 \\
\hline Diabetes type & & & 0.54 \\
\hline Type I & $15.8 \%(24 / 152)$ & $11.9 \%(8 / 67)$ & \\
\hline Type II & $84.2 \%(128 / 152)$ & $88.1 \%(59 / 67)$ & \\
\hline Hypercholesterolemia & $75.0 \%(252 / 336)$ & $67.8 \%(97 / 143)$ & 0.12 \\
\hline Hypertension & $87.5 \%(294 / 336)$ & $80.4 \%(115 / 143)$ & 0.049 \\
\hline Carotid artery disease & $19.0 \%(64 / 336)$ & $18.9 \%(27 / 143)$ & $>0.99$ \\
\hline Renal disease & $9.5 \%(32 / 336)$ & $11.9 \%(17 / 143)$ & 0.51 \\
\hline Pulmonary disease & $18.5 \%(62 / 336)$ & $14.7 \%(21 / 143)$ & 0.36 \\
\hline Congestive heart failure & $11.3 \%(38 / 336)$ & $11.2 \%(16 / 143)$ & $>0.99$ \\
\hline Previous cardiac arrhythmia & $9.8 \%(33 / 336)$ & $16.1 \%(23 / 143)$ & 0.06 \\
\hline Previous MI & $20.5 \%(69 / 336)$ & $15.4 \%(22 / 143)$ & 0.21 \\
\hline Smoking status & & & 0.54 \\
\hline Never & $14.6 \%(49 / 336)$ & $15.4 \%(22 / 143)$ & \\
\hline Quit & $54.2 \%(182 / 336)$ & $51.7 \%(74 / 143)$ & \\
\hline Still smokes & $31.3 \%(105 / 336)$ & $32.2 \%(46 / 143)$ & \\
\hline Unknown & $0 \%(0 / 336)$ & $0.7 \%(1 / 143)$ & \\
\hline Rutherford $0-3$ & $92.0 \%(309 / 336)$ & $89.4 \%(126 / 141)$ & 0.38 \\
\hline Rutherford 4-6 & $8.0 \%(27 / 336)$ & $10.6 \%(15 / 141)$ & \\
\hline Lesion length (mm) & $55.8 \pm 41.1(354)$ & $51.8 \pm 39.8(149)$ & 0.32 \\
\hline Total occlusion & $31.1 \%(110 / 354)$ & $28.2 \%(42 / 149)$ & 0.60 \\
\hline Proximal RVD (mm) & $5.1 \pm 1.0(350)$ & $5.0 \pm 0.9(148)$ & 0.65 \\
\hline Distal RVD (mm) & $5.0 \pm 1.0(350)$ & $5.0 \pm 1.1(148)$ & 0.98 \\
\hline MLD in lesion (mm) & $1.0 \pm 0.9(350)$ & $1.1 \pm 0.9(148)$ & 0.48 \\
\hline Percent diameter stenosis $(\%)$ & $79.5 \pm 17.1(350)$ & $78.5 \pm 16.7(148)$ & 0.59 \\
\hline Calcification & & & 0.08 \\
\hline None & $2.8 \%(10 / 354)$ & $5.4 \%(8 / 149)$ & \\
\hline Little & $30.2 \%(107 / 354)$ & $38.3 \%(57 / 149)$ & \\
\hline Moderate & $30.5 \%(108 / 354)$ & $22.1 \%(33 / 149)$ & \\
\hline Severe & $36.4 \%(129 / 354)$ & $34.2 \%(51 / 149)$ & \\
\hline Other stenosis in artery & & & 0.049 \\
\hline None & $51.1 \%(180 / 352)$ & $50.3 \%(75 / 149)$ & \\
\hline$\leq 50 \%$ & $32.1 \%(113 / 352)$ & $40.3 \%(60 / 149)$ & \\
\hline$>50 \%$ & $16.8 \%(59 / 352)$ & $9.4 \%(14 / 149)$ & \\
\hline Inflow tract stenosis & & & 0.023 \\
\hline None & $41.5 \%(146 / 352)$ & $47.0 \%(70 / 149)$ & \\
\hline$\leq 50 \%$ & $39.5 \%(139 / 352)$ & $43.6 \%(65 / 149)$ & \\
\hline$>50 \%$ & $19.0 \%(67 / 352)$ & $9.4 \%(14 / 149)$ & \\
\hline
\end{tabular}

Numbers in parentheses represent number of patients or number of lesions as appropriate 


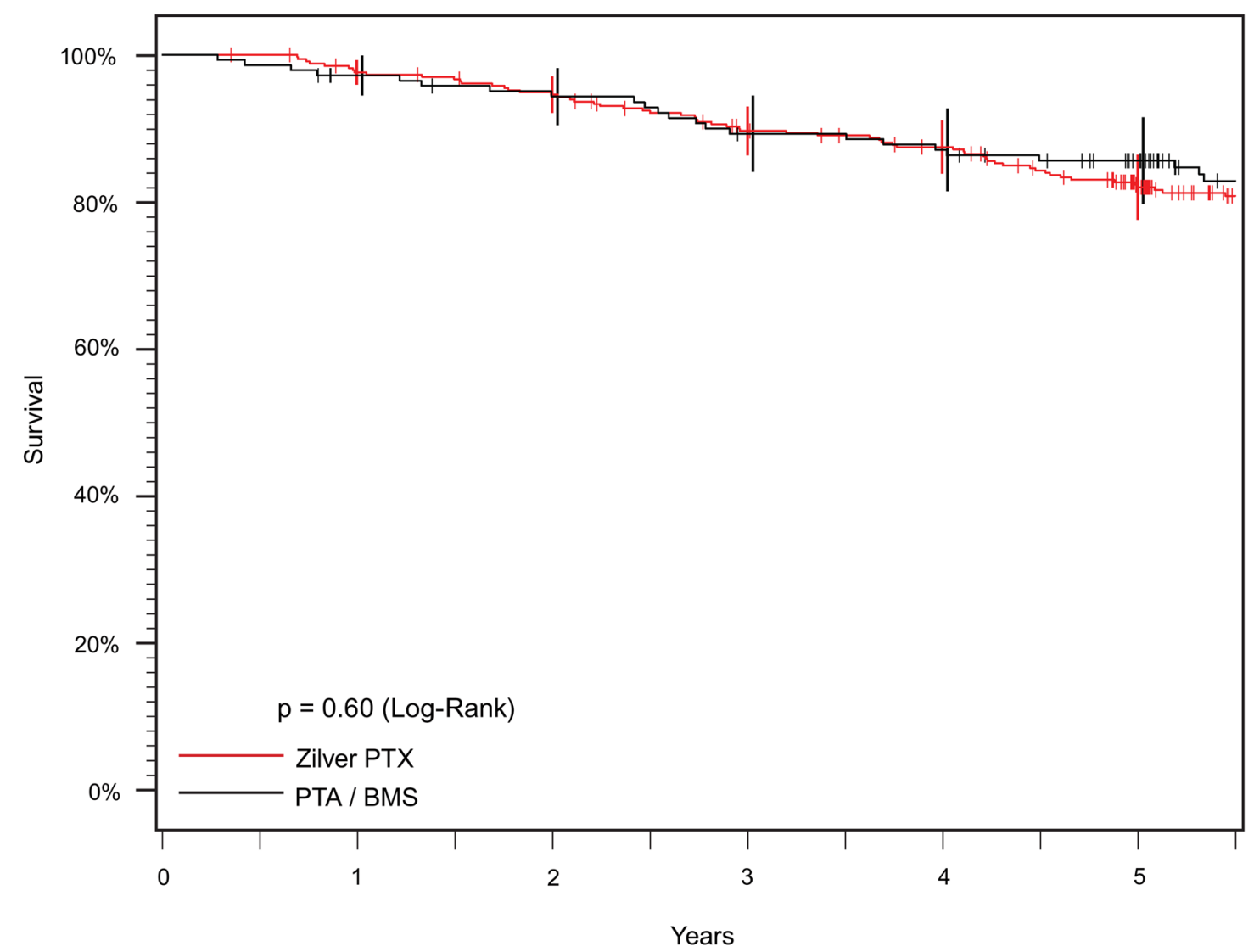

\begin{tabular}{|c|c|c|c|c|c|c|c|c|c|c|}
\hline \multirow{2}{*}{ Years } & \multicolumn{2}{|c|}{ Mortality Estimate } & \multicolumn{2}{c|}{ Standard Error } & \multicolumn{2}{c|}{ Failed } & \multicolumn{2}{c|}{ Censored } & \multicolumn{2}{c|}{ Remaining } \\
\cline { 2 - 12 } & $\mathbf{D E S}$ & $\begin{array}{c}\text { PTA / } \\
\text { BMS }\end{array}$ & DES & $\begin{array}{c}\text { PTA / } \\
\text { BMS }\end{array}$ & DES & $\begin{array}{c}\text { PTA / } \\
\text { BMS }\end{array}$ & DES & $\begin{array}{c}\text { PTA / } \\
\text { BMS }\end{array}$ & $\begin{array}{c}\text { DES } \\
\text { PTA / } \\
\text { BMS }\end{array}$ \\
\hline $\mathbf{0}$ & $0.0 \%$ & $0.0 \%$ & $0.0 \%$ & $0.0 \%$ & 0 & 0 & 0 & 0 & 336 & 143 \\
\hline $\mathbf{1}$ & $2.4 \%$ & $2.8 \%$ & $0.8 \%$ & $1.4 \%$ & 8 & 4 & 3 & 3 & 325 & 136 \\
\hline $\mathbf{2}$ & $5.4 \%$ & $5.7 \%$ & $1.2 \%$ & $2.0 \%$ & 18 & 8 & 5 & 4 & 313 & 131 \\
\hline $\mathbf{3}$ & $10.3 \%$ & $10.7 \%$ & $1.7 \%$ & $2.6 \%$ & 34 & 15 & 12 & 5 & 290 & 123 \\
\hline $\mathbf{4}$ & $12.5 \%$ & $12.9 \%$ & $1.9 \%$ & $2.9 \%$ & 41 & 18 & 17 & 5 & 278 & 120 \\
\hline $\mathbf{5}$ & $18.0 \%$ & $14.3 \%$ & $2.2 \%$ & $3.0 \%$ & 58 & 20 & 40 & 15 & 238 & 108 \\
\hline Final & $19.1 \%$ & $17.1 \%$ & $2.5 \%$ & $3.6 \%$ & 61 & 23 & - & - & - & - \\
\hline
\end{tabular}

Fig. 2 Kaplan-Meier survival analysis for patients in the Zilver PTX RCT. Long-term mortality analysis shows no difference between the DES (red curve) and PTA/BMS (black curve). RCT, randomized

were 22 deaths through 3 years. Through 3 years, the risk of mortality was $15.8 \%$ for DES group and $15.3 \%$ for BMS group. Through 5 years, the risk of mortality was $25.9 \%$ for the DES group. There was no difference in mortality between the two groups (log-rank $p=0.89$; Fig. 4).

The Cox proportional hazards model revealed that critical limb ischemia (CLI, $p<0.001)$, age $(p<0.001)$, renal failure $(p<0.001)$, and gender $(p=0.001)$ were significantly associated with mortality (Fig. 5). Hypercholesterolemia $(p=0.004)$ was associated with lower risk of mortality. Treatment with Zilver PTX $(p=0.49)$ was not associated with mortality. In the covariate analysis of controlled trial; DES, drug-eluting stent; PTA, percutaneous transluminal angioplasty; BMS, bare metal stent

paclitaxel dose, the significant factors were identical to the treatment analysis, and there was no association or trend of paclitaxel dose ( $p=0.07)$ with mortality (Online Resource 4). Table 2 shows the causes of death in the DES group through 5 years.

\section{Discussion}

Recently, Katsanos et al. [12] published a meta-analysis of 28 RCTs (24 DCB and 4 DES) that raised concerns regarding the long-term safety of paclitaxel-coated devices. 
Table 2 Causes of death through 5 years

\begin{tabular}{lllll}
\hline Cause & \multicolumn{2}{l}{ Zilver PTX RCT } & & \multicolumn{2}{l}{\begin{tabular}{l} 
Zilver PTX Japan PMS \\
\cline { 2 - 3 }
\end{tabular}} & DES & PTA/BMS & $p$ value & DES \\
\hline Cardiovascular & $4.8 \%(16 / 336)$ & $5.6 \%(8 / 143)$ & 0.66 & $6.1 \%(55 / 904)$ \\
Cancer & $4.8 \%(16 / 336)$ & $1.4 \%(2 / 143)$ & 0.11 & $3.0 \%(27 / 904)$ \\
Pulmonary & $1.8 \%(6 / 336)$ & $1.4 \%(2 / 143)$ & $>0.99$ & $2.7 \%(24 / 904)$ \\
Stroke & $0.6 \%(2 / 336)$ & $0.7 \%(1 / 143)$ & $>0.99$ & $1.5 \%(14 / 904)$ \\
Trauma/accident & $0.0 \%(0 / 336)$ & $1.4 \%(2 / 143)$ & 0.09 & $0.2 \%(2 / 904)$ \\
GI & $0.3 \%(1 / 336)$ & $0.0 \%(0 / 143)$ & $>0.99$ & $0.2 \%(2 / 904)$ \\
Infection & $0.0 \%(0 / 336)$ & $0.0 \%(0 / 143)$ & $>0.99$ & $0.2 \%(2 / 904)$ \\
Renal & $0.0 \%(0 / 336)$ & $0.0 \%(0 / 143)$ & $>0.99$ & $0.8 \%(7 / 904)$ \\
Multiple & $0.3 \%(1 / 336)$ & $0.7 \%(1 / 143)$ & 0.51 & $1.5 \%(14 / 904)$ \\
Unknown & $1.8 \%(6 / 336)$ & $0.7 \%(1 / 143)$ & 0.68 & $4.3 \%(39 / 904)$ \\
\hline
\end{tabular}

${ }^{a}$ Cause of death was only available for the 65 deaths reported during active study follow-up

Tissue Loss
Congestive Heart Failure
Still Smokes vs Never Smoked
Renal Disease
Arrhythmia
Pulmonary Disease
Carotid Disease
Diabetes Mellitus
Quit Smoking vs Never Smoked
CLI vs Claudicant
US vs Japan
US vs Germany
Hypercholesterolemia
Zilver PTX
Male vs Female
Hypertension
Age (yr)
Total Lesion Length (cm)
Body Mass Index
Previous Myocardial Infarction

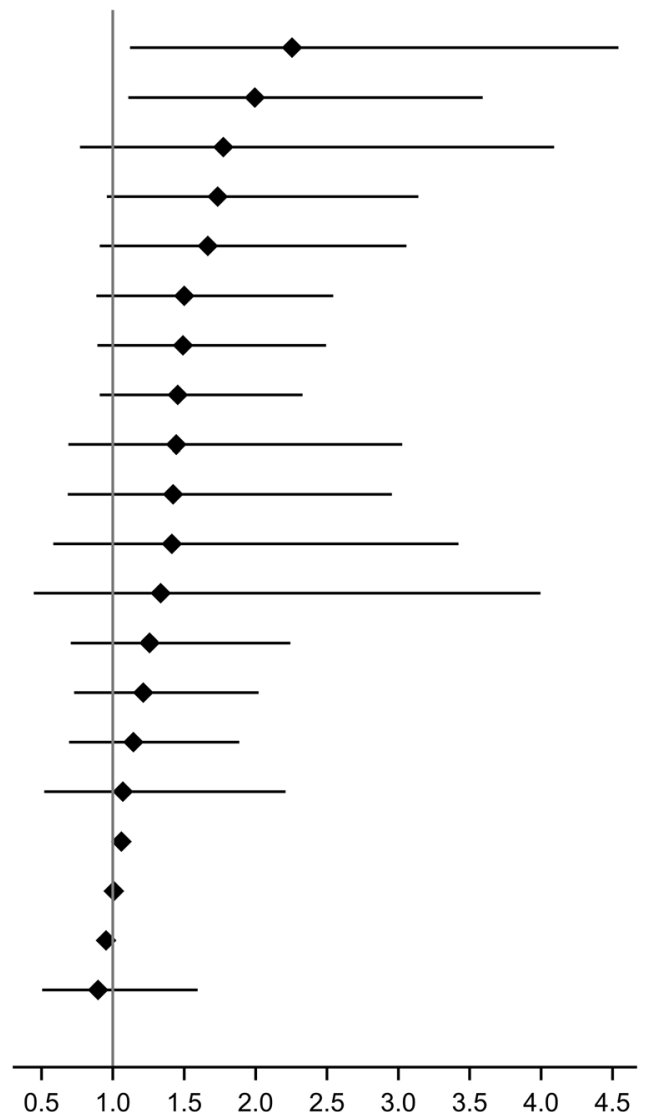

Hazard Ratio

$2.256(1.120,4.542)$

$1.995(1.108,3.591)$

0.021

$1.774(0.769,4.092)$

0.403

$1.735(0.958,3.140) \quad 0.069$

$1.665(0.908,3.056) \quad 0.100$

$1.500(0.884,2.544) \quad 0.133$

$1.491(0.891,2.493) \quad 0.128$

$1.454(0.908,2.329) \quad 0.119$

$1.444(0.689,3.027) \quad 0.403$

$1.422(0.684,2.955) \quad 0.345$

$1.413(0.583,3.422) \quad 0.689$

$1.335(0.446,3.996) \quad 0.689$

$1.257(0.705,2.244) \quad 0.438$

$1.213(0.728,2.021) \quad 0.458$

$1.143(0.693,1.886) \quad 0.600$

$1.071(0.519,2.210) \quad 0.853$

$1.060(1.029,1.092) \quad<.001$

$1.006(0.958,1.057) \quad 0.807$

$0.952(0.905,1.002) \quad 0.060$

$0.897(0.505,1.594) \quad 0.711$

Fig. 3 Covariate analysis for treatment in the Zilver PTX RCT. Cox proportional hazards model for mortality through 5 years. The diamonds indicate the hazard ratios, and the lines indicate the $95 \%$ confidence intervals. RCT, randomized controlled trial

The findings have undoubtedly had a major impact on the clinical management of patients with PAD, specifically regarding the choice of therapies for lower extremity revascularization $[15,16]$. The included RCTs varied in patient populations, with potentially unequal comorbidities, as well as differences in control group therapies.
Although the meta-analysis pooled results for DES and DCB, there are important device-specific differences. The Zilver PTX DES has a similar paclitaxel dose compared to other peripheral paclitaxel DES and low dose compared to DCB. The total amount of paclitaxel on each device is dependent on device dimensions and the surface area 
Table 3 Japan PMS demographics and baseline lesion characteristics

\begin{tabular}{|c|c|c|c|}
\hline Characteristic & DES & BMS & $p$ value \\
\hline Age (years) & $73.5 \pm 8.5(904)$ & $73.8 \pm 8.7(190)$ & 0.68 \\
\hline Gender & & & 0.79 \\
\hline Male & $70.2 \%(635 / 904)$ & $71.6 \%(136 / 190)$ & \\
\hline Female & $29.8 \%(269 / 904)$ & $28.4 \%(54 / 190)$ & \\
\hline Diabetes & $58.7 \%(531 / 904)$ & $48.9 \%(93 / 190)$ & 0.015 \\
\hline Diabetes type & & & 0.28 \\
\hline Type I & $8.3 \%(43 / 518)$ & $4.4 \%(4 / 90)$ & \\
\hline Type II & $91.7 \%(475 / 518)$ & $95.6 \%(86 / 90)$ & \\
\hline Hypercholesterolemia & $61.0 \%(551 / 904)$ & $50.0 \%(95 / 190)$ & 0.006 \\
\hline Hypertension & $85.5 \%(773 / 904)$ & $78.4 \%(149 / 190)$ & 0.021 \\
\hline Carotid disease & $58.3 \%(527 / 904)$ & $45.3 \%(86 / 190)$ & 0.001 \\
\hline Renal disease & $43.6 \%(394 / 904)$ & $40.5 \%(77 / 190)$ & 0.47 \\
\hline Chronic renal failure & $35.8 \%(323 / 903)$ & $34.7 \%(66 / 190)$ & 0.80 \\
\hline Pulmonary disease & $7.5 \%(68 / 904)$ & $6.8 \%(13 / 190)$ & 0.88 \\
\hline Smoking status & & & 0.11 \\
\hline Never & $36.5 \%(330 / 904)$ & $33.2 \%(63 / 190)$ & \\
\hline Quit & $45.0 \%(407 / 904)$ & $41.6 \%(79 / 190)$ & \\
\hline Still smokes & $18.5 \%(167 / 904)$ & $25.3 \%(48 / 190)$ & \\
\hline Rutherford 0-3 & $77.9 \%(663 / 851)$ & $65.7 \%(115 / 175)$ & $<0.001$ \\
\hline Rutherford 4-6 & $22.1 \%(188 / 851)$ & $34.3 \%(60 / 175)$ & \\
\hline Lesion length (mm) & $14.6 \pm 9.6(1088)$ & $11.1 \pm 8.4(202)$ & $<0.001$ \\
\hline Total occlusion & $41.3 \%(450 / 1089)$ & $35.1 \%(71 / 202)$ & 0.10 \\
\hline $\mathrm{RVD}(\mathrm{mm})$ & $5.7 \pm 0.9(1088)$ & $5.7 \pm 0.9(202)$ & 0.77 \\
\hline Percent diameter stenosis (\%) & $91.7 \pm 10.8(1089)$ & $91.6 \pm 10.1(202)$ & 0.93 \\
\hline Calcification & & & 0.021 \\
\hline None & $26.8 \%(292 / 1089)$ & $29.2 \%(59 / 202)$ & \\
\hline Little & $34.9 \%(380 / 1089)$ & $41.6 \%(84 / 202)$ & \\
\hline Moderate & $21.0 \%(229 / 1089)$ & $19.8 \%(40 / 202)$ & \\
\hline Severe & $17.3 \%(188 / 1089)$ & $9.4 \%(19 / 202)$ & \\
\hline Patent runoff vessels & & & 0.34 \\
\hline 0 & $6.6 \%(71 / 1083)$ & $10.0 \%(20 / 201)$ & \\
\hline 1 & $31.9 \%(345 / 1083)$ & $31.3 \%(63 / 201)$ & \\
\hline 2 & $32.7 \%(354 / 1083)$ & $29.4 \%(59 / 201)$ & \\
\hline 3 & $28.9 \%(313 / 1083)$ & $29.4 \%(59 / 201)$ & \\
\hline In-stent restenosis & $18.9 \%(206 / 1089)$ & $7.9 \%(16 / 202)$ & $<0.001$ \\
\hline
\end{tabular}

Numbers in parentheses represent number of patients or number of lesions as appropriate coated; specific doses are provided in manufacturer instructions for use. Additionally, the coating consists of paclitaxel only and is free of any polymer or excipient [17]. Therefore, after drug elution is complete, only a BMS remains, with no long-term paclitaxel exposure.

The meta-analysis was also limited to published reports of trial outcomes, which do not provide individual patientlevel information regarding cause-specific mortality data, total paclitaxel dose delivered, follow-up, or exposure to paclitaxel from contralateral endovascular treatment of PAD or protocol-defined re-intervention. These limitations bring into question the poolability of the RCTs and have the potential to alter the findings of the meta-analysis.

The previously published Zilver PTX RCT data reported 5-year all-cause mortality for only the primary randomization intent-to-treat groups [5]. However, $40 \%$ of the patients included in the PTA group in that mortality analysis were actually treated with the DES as part of protocoldefined secondary randomization and crossover. Therefore, conclusions regarding a potential association of paclitaxel with long-term mortality based on these results are flawed. This highlights the importance of relying on patient-level data rather than summary data, a limitation of the meta- 


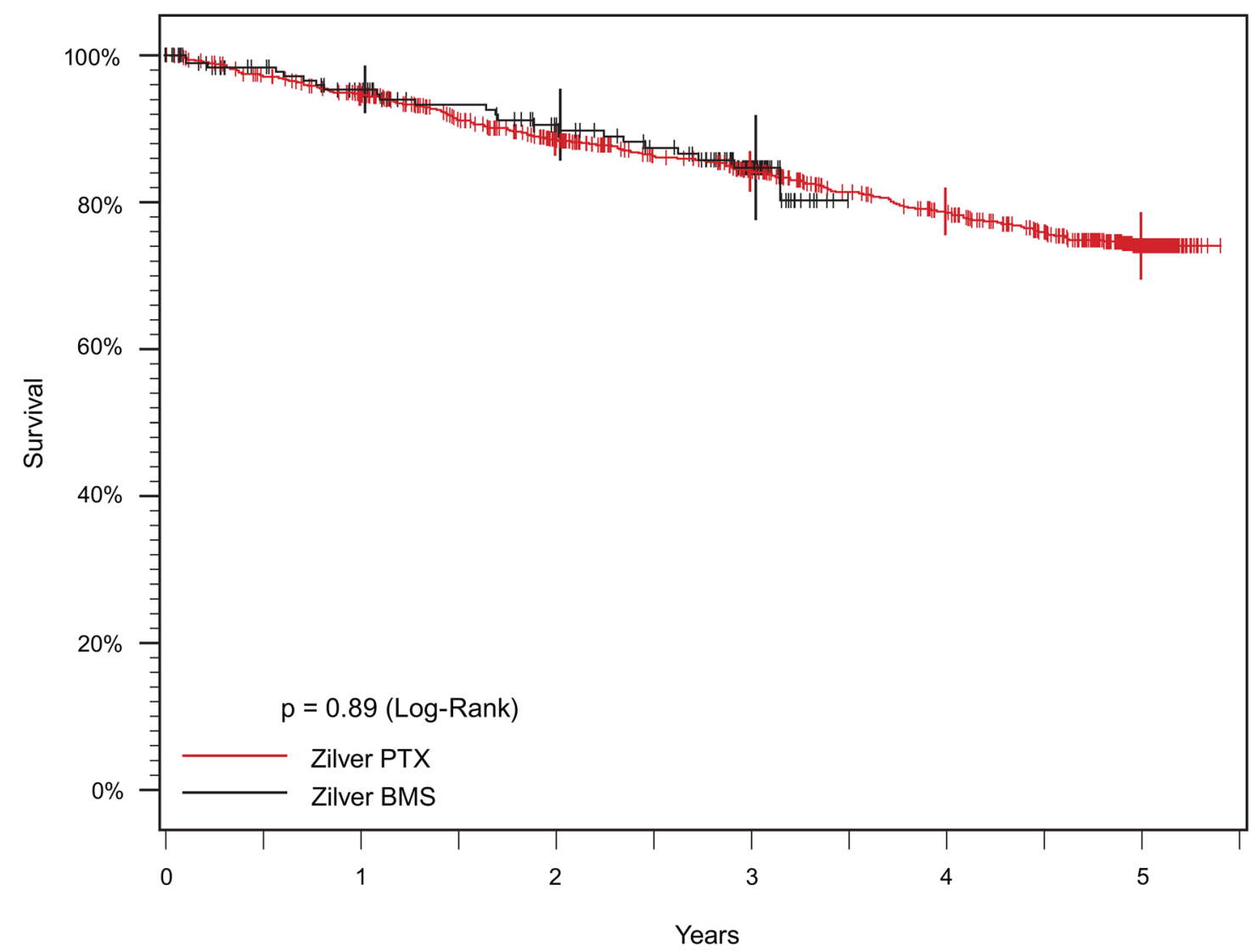

\begin{tabular}{|c|c|c|c|c|c|c|c|c|c|c|}
\hline \multirow{2}{*}{ Years } & \multicolumn{2}{|c|}{ Mortality Estimate } & \multicolumn{2}{|c|}{ Standard Error } & \multicolumn{2}{|c|}{ Failed } & \multicolumn{2}{|c|}{ Censored } & \multicolumn{2}{|c|}{ Remaining } \\
\hline & DES & BMS & DES & BMS & DES & BMS & DES & BMS & DES & BMS \\
\hline 0 & $0.0 \%$ & $0.0 \%$ & $0.0 \%$ & $0.0 \%$ & 0 & 0 & 0 & 0 & 904 & 190 \\
\hline 1 & $5.4 \%$ & $4.7 \%$ & $0.8 \%$ & $1.6 \%$ & 47 & 8 & 48 & 29 & 809 & 153 \\
\hline 2 & $11.4 \%$ & $9.5 \%$ & $1.1 \%$ & $2.5 \%$ & 96 & 15 & 132 & 52 & 676 & 123 \\
\hline 3 & $15.8 \%$ & $15.3 \%$ & $1.4 \%$ & $3.6 \%$ & 127 & $22^{*}$ & 211 & 108 & 566 & 60 \\
\hline 4 & $21.3 \%$ & & $1.7 \%$ & & 161 & & 275 & & 468 & \\
\hline 5 & $25.9 \%$ & & $2.3 \%$ & & 186 & & 502 & & 216 & \\
\hline
\end{tabular}

* One additional event occurred after 3 years (1095 days)

Fig. 4 Kaplan-Meier survival analysis for patients in the Japan PMS. Long-term mortality analysis shows no difference between the DES (red curve) and BMS (black curve). PMS, post-market study; DES, drug-eluting stent; BMS, bare metal stent

analysis that has been noted by others [18-20]. Additionally, missing data for patients who did not complete the study were a limitation of previous analyses. Vital status data are now available for $94 \%$ of the patients to address this limitation.

The RCT protocol allowed placement of the DES for treatment of procedural PTA failure or for re-intervention of non-paclitaxel-treated lesions within the first year. As a result, 63 PTA patients were treated with the DES at the time of the procedure. An additional 31 patients failed their initial non-paclitaxel therapy and crossed over to treatment with DES at a median of 183 days. As defined in the study protocol, these crossover patients were then followed for the remaining 4-5 years of the study as DES patients. The published meta-analysis includes patients secondarily treated with paclitaxel devices in the PTA control group; this fundamental flaw confounds the interpretation of the results.

When evaluating the association of a drug or device with increased mortality, the most appropriate group of patients to analyze should be considered. This approach is supported by internationally harmonized guidelines endorsed by numerous regulatory bodies that state, "For the overall safety and tolerability assessment, the set of subjects to be summarized is usually defined as those subjects who received at least one dose of the investigational drug" [21]. Analyses presented in RCTs favor a conservative intent-to-treat approach to evaluate 


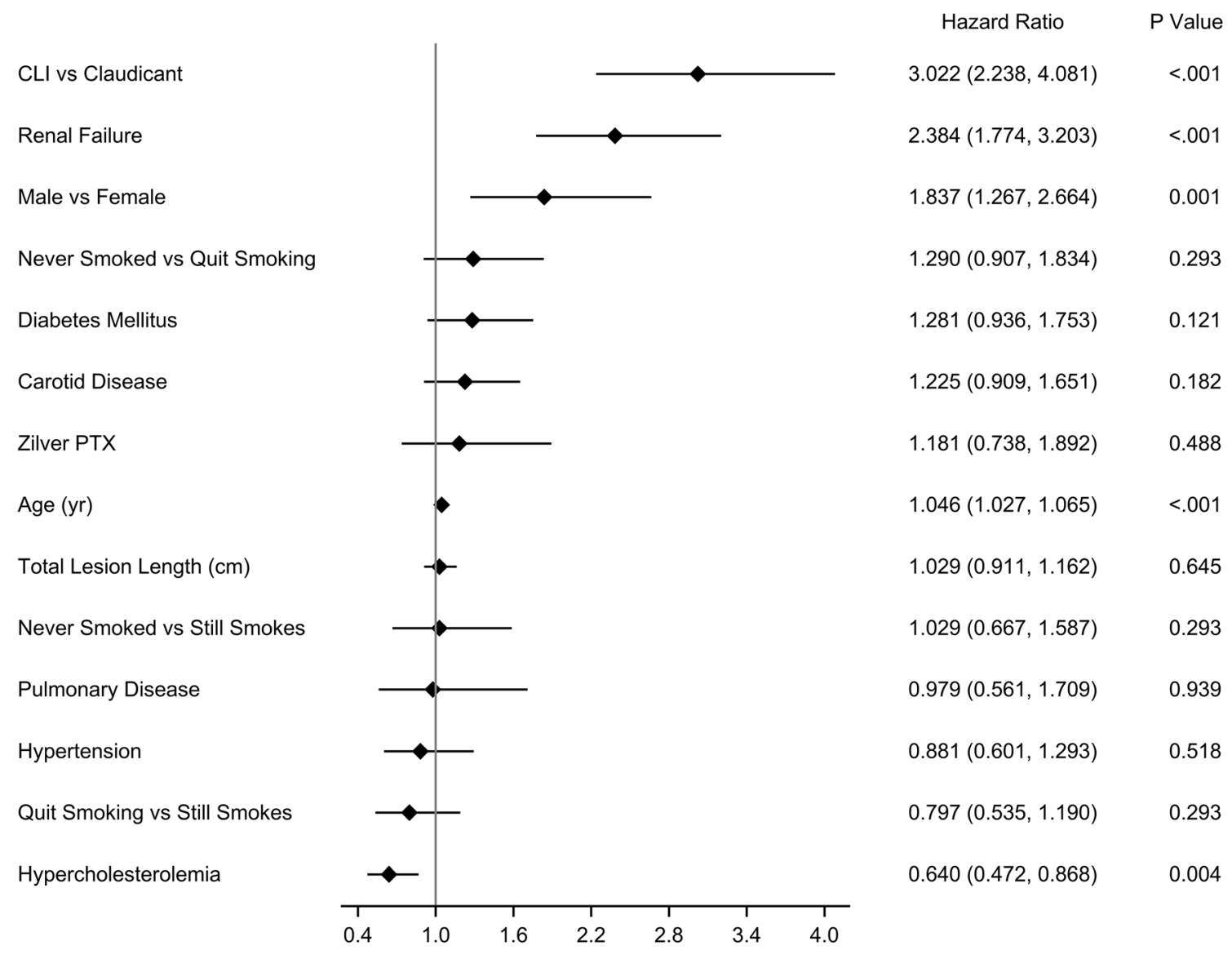

Fig. 5 Covariate analysis for treatment in Japan PMS. Cox proportional hazards model for mortality through 5 years. The diamonds indicate the hazard ratios, and the lines indicate the $95 \%$ confidence intervals. PMS, post-market study

effectiveness. However, this approach cannot be used to analyze safety in this case because of a protocol-defined secondary randomization and crossover to treatment with DES, which together accounts for $40 \%$ of the patients originally randomized to PTA. The appropriate analysis compares patients who actually received the DES to patients who were not treated with the DES during the study; the analyses in this paper are consistent with these recommendations to evaluate actual treatment. The criticism that this approach results in a non-randomized comparison can be accounted for by using statistical methods such as a Cox proportional hazards model.

To address the limitation of the non-randomized comparison of DES and PTA/BMS patients, we sought to identify which factors were associated with mortality while also accounting for potential differences between patient characteristics. As expected, age and other comorbidities common in PAD patients were significantly associated with an increased risk of mortality, whereas treatment with the DES was not associated with an increased mortality risk in either the RCT or Japan PMS.

Dose escalation studies have shown that increasing dose corresponds to an increased response [22]. The authors of the recent meta-analysis proposed a relationship between paclitaxel dose and excess mortality [12]. A retrospective study revealed that mortality risk following application of the DES did not increase over 5 years, irrespective of the paclitaxel dose [23]. Despite the very low dose and transient systemic and tissue levels of paclitaxel following DES implantation, a Cox model that included total paclitaxel dose received by the patient was performed. In this analysis, the significant factors associated with an increased risk of mortality were identical to the factors identified in the treatment analysis. In both the RCT and Japan PMS, paclitaxel dose was not significantly associated with mortality. Therefore, a relationship between paclitaxel and late mortality cannot be deemed causative. Due to the post-market design of the Japan PMS, more limited data were collected on patient demographics and comorbidities than may be collected in a pre-market study. As a result, important factors relating to long-term patient mortality across a broad patient population may not have been fully identified.

In comparison with another study that enrolled Japanese PAD patients [24], the mortality rate through 5 years in the Japan DES PMS was comparable despite enrolling patients 
with more severe comorbidities, suggesting that mortality is a result of the natural progression of PAD and not any paclitaxel-specific effect. Although the mortality rate in the Japan DES and BMS PMS was higher than in the RCT, the RCT included a limited patient population. In clinical practice, patients with a wide range of disease are treated; therefore, the safety information from the large Japan PMS, that showed no difference for paclitaxel and non-paclitaxel-eluting devices, is more representative of patients treated in standard clinical practice.

The TransAtlantic Inter-Society Consensus (TASC) document reports a 5-year all-cause mortality rate of approximately $30 \%$ for patients with intermittent claudication [25]. Similarly, a retrospective review of intermittent claudicants who underwent endovascular revascularization reported a 5-year all-cause mortality rate of 24\% [26]. Heikkila et al. [27] reported a 5-year all-cause mortality rate of $24.7 \%$ for 26,579 patients with intermittent claudication who underwent endovascular revascularization. In a meta-analysis by Sigvant et al. [28], the authors reported a 5-year all-cause mortality rate of $27 \%$ for 57,322 patients with symptomatic PAD (90\% with intermittent claudication, $10 \%$ with CLI). Secemsky et al. [29] recently published a report that evaluated 51,456 patients in a US Centers for Medicare and Medicaid Services database who underwent peripheral artery stenting; $59.7 \%$ had CLI. Through 4.1 years, the mortality rate for patients treated with DES was not significantly different compared to patients treated with BMS $(51.7 \%$ vs. $50.1 \%)$. The mortality rate in the Japan PMS, which included $22 \%$ of patients with CLI, was similar to the published rates and may be a reliable indicator of long-term mortality rates in the PAD patient population. The lower mortality rate in the RCT may reflect the more controlled clinical study environment.

In the Zilver PTX RCT, the most prevalent causes of death were cardiovascular disease and cancer. Although the rate of cardiovascular disease-related deaths was higher in the PTA/BMS group and the rate of cancer-related deaths was higher in the DES group, neither of these differences was significant. McDermott et al. [30] reported a 3-year cancer mortality rate of $3.0 \%$ in a cohort of 1314 PAD patients, corresponding to a 1-year annualized rate of $1.0 \%$ (i.e., approximately 5\% through 5 years). Rantner et al. [31] reported that cancer was the leading cause of death in a population of 255 intermittent claudication patients, occurring in $3.9 \%$ of patients $(10 / 255)$ within 5 years. These published rates for cancer-related mortality in PAD patients are comparable to the rates seen in the Zilver PTX RCT $(4.8 \%)$ and Japan PMS (3.0\%) studies through 5 years.

As discussed, the patient populations from the RCT and Japan PMS studies are different. Therefore, these data could not be pooled for analysis. When evaluated independently, neither study shows a significant increase in mortality for patients treated with the DES versus those not treated with the DES.

Unlike the Japan DES PMS, the Japan BMS PMS was limited to 3-year follow-up. However, there was no difference in mortality between DES and BMS patients. Both groups have an approximately 5\% annual mortality rate over the available follow-up periods with no apparent increase in the annual mortality rate beyond 3 years with the DES.

During enrollment and follow-up of the RCT and Japan PMS, other peripheral paclitaxel devices were not available in the USA or Japan. Therefore, the potential confounding effect of paclitaxel use in the control group for re-intervention or treatment of the contralateral leg beyond the study protocol is minimal for these studies.

In summary, analyses of the paclitaxel-coated Zilver PTX DES utilizing patient-level data from the Zilver PTX RCT and Japan PMS demonstrated no increase in longterm all-cause mortality. Neither of these datasets substantiated the signal described by Katsanos et al. [12].

Funding These studies were funded by Cook Medical.

\section{Compliance with Ethical Standards}

Conflict of interest MDD and GMA are paid consultants for Cook Medical. AH is a Medical Advisory Board member for Boston Scientific Corp., Gore \& Associates and Medtronic, and is a Clinical Investigator for Cook Medical, Shockwave, Intact Vascular, Endologix, Philips. MRJ is a non-compensated advisor for Abbott Vascular, Boston Scientific, and a compensated advisor for Biotronik, Medtronic, Philips/Volcano, Micell, Vactronix, Sanofi; Equity Investor: Embolitech, Gemini, PQ Bypass, and Vascular Therapies. AEL, EEO'L, and ATS are paid employees of Cook Medical. MS is a paid consultant for Abbott, Cook, Endologix, Medtronic, Philips, and Silk Road. HY has received honoraria from Boston Scientific Corp, Cook Medical, and Terumo. TZ has received honoraria from Abbott Vascular, Veryan, Biotronik, Boston Scientific Corp., Cook Medical, Gore \& Associates, Medtronic, Philips-Spectranetics, Shockwave; consulted for Boston Scientific Corp., Gore \& Associates, Medtronic, Veryan, Intact Vascular, Shockwave, Bayer, Vesper Medical; received (institution) research, clinical trial, or drug study funds from 480 biomedical, Bard Peripheral Vascular, Veryan, Biotronik, Cook Medical, Gore \& Associates, Medtronic, Philips, Terumo, TriReme, Shockwave, Med Alliance, Intact Vascular, B. Braun, and has common stock in QT Medical. MB and OI report no relevant conflicts to disclose.

Ethical Approval All procedures performed in studies involving human participants were in accordance with the ethical standards of the institutional and/or national research committee and with the 1964 Declaration of Helsinki and its later amendments or comparable ethical standards.

Informed Consent For the Zilver PTX RCT, informed consent was obtained from all individual participants included in the study. For the Japan DES PMS and Japan BMS PMS studies, informed consent 
processes were determined by each institution's ethical committee policy to specify whether informed consent was necessary or outcome data could be abstracted while protecting patients' rights without requiring individual patient consent.

Open Access This article is distributed under the terms of the Creative Commons Attribution 4.0 International License (http:// creativecommons.org/licenses/by/4.0/), which permits unrestricted use, distribution, and reproduction in any medium, provided you give appropriate credit to the original author(s) and the source, provide a link to the Creative Commons license, and indicate if changes were made.

\section{References}

1. Society for Vascular Surgery Lower Extremity Guidelines Writing Group, Conte MS, Pomposelli FB, Clair DG, Geraghty PJ, McKinsey JF, et al. Society for Vascular Surgery practice guidelines for atherosclerotic occlusive disease of the lower extremities management of asymptomatic disease and claudication. J Vasc Surg. 2015;61(3 Suppl):2S-41S. https://doi.org/10. 1016/j.jvs.2014.12.009.

2. Aboyans V, Ricco JB, Bartelink MEL, Bjorck M, Brodmann M, Cohnert T, et al. 2017 ESC Guidelines on the diagnosis and treatment of peripheral arterial diseases, in collaboration with the European Society for Vascular Surgery (ESVS): document covering atherosclerotic disease of extracranial carotid and vertebral, mesenteric, renal, upper and lower extremity arteries. Eur Heart J. 2018;39(9):763-816. https://doi.org/10.1093/eurheartj/ehx095.

3. Dake MD, Ansel GM, Jaff MR, Ohki T, Saxon RR, Smouse HB, et al. Paclitaxel-eluting stents show superiority to balloon angioplasty and bare metal stents in femoropopliteal disease: twelve-month Zilver PTX randomized study results. Circ Cardiovasc Interv. 2011;4(5):495-504. https://doi.org/10.1161/ circinterventions.111.962324.

4. Dake MD, Ansel GM, Jaff MR, Ohki T, Saxon RR, Smouse HB, et al. Sustained safety and effectiveness of paclitaxel-eluting stents for femoropopliteal lesions: 2-year follow-up from the Zilver PTX randomized and single-arm clinical studies. J Am Coll Cardiol. 2013;61(24):2417-27. https://doi.org/10.1016/j. jacc.2013.03.034.

5. Dake MD, Ansel GM, Jaff MR, Ohki T, Saxon RR, Smouse HB, et al. Durable clinical effectiveness with paclitaxel-eluting stents in the femoropopliteal artery: 5-year results of the Zilver PTX randomized trial. Circulation. 2016;133(15):1472-83. https://doi. org/10.1161/circulationaha.115.016900.

6. Ansel GM, Jaff MR, Popma JJ, Battisti AJ, Lottes AE, Harnish P, et al. A quantitative angiographic comparison of restenotic tissue following placement of drug-eluting stents and bare metal stents in symptomatic patients with femoropopliteal disease. J Endovasc Ther. 2017;24(4):499-503. https://doi.org/10.1177/152660281 7708778 .

7. Tepe G, Schnorr B, Albrecht T, Brechtel K, Claussen CD, Scheller B, et al. Angioplasty of femoral-popliteal arteries with drug-coated balloons: 5-year follow-up of the THUNDER trial. J Am Coll Cardiol Interv. 2015;8:102-8. https://doi.org/10.1016/ j.cin.2014.07.023.

8. Brodmann M, Werner M, Meyer DR, Reimer P, Kruger K, Granada JF, et al. Sustainable antirestenosis effect with a lowdose drug-coated balloon: the ILLUMENATE European randomized clinical trial 2-year results. JACC Cardiovasc Interv. 2018;11(23):2357-64. https://doi.org/10.1016/j.jcin.2018.08.034.
9. Rosenfield K, Jaff MR, White CJ, Rocha-Singh K, Mena-Hurtado C, Metzger DC, et al. Trial of a paclitaxel-coated balloon for femoropopliteal artery disease. N Engl J Med. 2015;373(2):145-53. https://doi.org/10.1056/NEJMoa1406235.

10. Tepe G, Laird J, Schneider P, Brodmann M, Krishnan P, Micari $A$, et al. Drug-coated balloon versus standard percutaneous transluminal angioplasty for the treatment of superficial femoral and popliteal peripheral artery disease: 12-month results from the IN.PACT SFA randomized trial. Circulation. 2015;131(5):495-502. https://doi.org/10.1161/circulationaha.114. 011004.

11. Steiner S, Willfort-Ehringer A, Sievert H, Geist V, Lichtenberg M, Del Giudice C, et al. 12-Month results from the first-in-human randomized study of the Ranger paclitaxel-coated balloon for femoropopliteal treatment. JACC Cardiovasc Interv. 2018;11(10):934-41. https://doi.org/10.1016/j.jcin.2018.01.276.

12. Katsanos K, Spiliopoulos S, Kitrou P, Krokidis M, Karnabatidis D. Risk of death following application of paclitaxel-coated balloons and stents in the femoropopliteal artery of the leg: a systematic review and meta-analysis of randomized controlled trials. J Am Heart Assoc. 2018;7(24):e011245. https://doi.org/10.1161/ jaha.118.011245.

13. Yokoi H, Ohki T, Kichikawa K, Nakamura M, Komori K, Nanto $\mathrm{S}$, et al. Zilver PTX post-market surveillance study of paclitaxeleluting stents for treating femoropopliteal artery disease in Japan: 12-month results. J Am Coll Cardiol Interv. 2016;9(3):271-7. https://doi.org/10.1016/j.jcin.2015.09.035.

14. Kichikawa K, Ichihashi S, Yokoi H, Ohki T, Nakamura M, Komori K, et al. Zilver PTX post-market surveillance study of paclitaxel-eluting stents for treating femoropopliteal artery disease in Japan: 2-year results. Cardiovasc Intervent Radiol. 2018. https://doi.org/10.1007/s00270-018-2110-1.

15. U.S. Food and Drug Administration. UPDATE: treatment of peripheral arterial disease with paclitaxel-coated balloons and paclitaxel-eluting stents potentially associated with increased mortality-letter to health care providers. 07 August 2019. https://www.fda.gov/medical-devices/letters-health-careproviders/August-7-2019-update-treatment-peripheral-arterialdisease-paclitaxel-coated-balloons-and-paclitaxel. Accessed 07 August 2019.

16. Cardiovascular and Interventional Radiological Society of Europe. CIRSE Position on the use of paclitaxel-coated balloons and stents in PAD. 01 July 2019. https://www.cirse.org/research/ current-updates/. Accessed 01 July 2019.

17. Dake MD, Van Alstine WG, Zhou Q, Ragheb AO. Polymer-free paclitaxel-coated Zilver PTX stents-evaluation of pharmacokinetics and comparative safety in porcine arteries. J Vasc Interv Radiol. 2011;22(5):603-10. https://doi.org/10.1016/j.jvir.2010. 12.027.

18. Schneider PA, Laird JR, Doros G, Gao Q, Ansel G, Brodmann M, et al. Mortality not correlated with paclitaxel exposure: an independent patient-level meta-analysis of a drug-coated balloon. J Am Coll Cardiol. 2019;73(20):2550-63. https://doi.org/10. 1016/j.jacc.2019.01.013.

19. Shishehbor MH, Secemsky EA, Varcoe RL. Is there a real association between paclitaxel devices and mortality? Time to pause and re-evaluate what we know about this statistical finding. J Am Heart Assoc. 2019;8(10):e012524. https://doi.org/10.1161/ jaha.119.012524.

20. Albrecht T, Schnorr B, Kutschera M, Waliszewski MW. Twoyear mortality after angioplasty of the femoro-popliteal artery with uncoated balloons and paclitaxel-coated balloons-a pooled analysis of four randomized controlled multicenter trials. Cardiovasc Intervent Radiol. 2019;42(7):949-55. https://doi.org/10. 1007/s00270-019-02194-w. 
21. U.S. Food and Drug Administration. International conference on harmonisation. E9 statistical principles for clinical trials. September 1998. https://www.fda.gov/downloads/drugs/guidance complianceregulatoryinformation/guidances/ucm073137. pdf. Accessed 10 May 2019.

22. Tallarida RJ, Jacob LS. The dose-response relation. The doseresponse relation in pharmacology. New York, NY: Springer US; 1979. p. 1-17.

23. Katsuki T, Takahara M, Soga Y, Okamoto S, Iida O, Fujihara M, et al. Mortality risk following application of a paclitaxel-coated stent in femoropopliteal lesions. J Endovasc Ther. 2019. https:// doi.org/10.1177/1526602819870309.

24. Soga Y, Iida O, Hirano K, Yokoi H, Nanto S, Nobuyoshi M. Midterm clinical outcome and predictors of vessel patency after femoropopliteal stenting with self-expandable nitinol stent. J Vasc Surg. 2010;52(3):608-15. https://doi.org/10.1016/j.jvs. 2010.03.050.

25. Norgren L, Hiatt WR, Dormandy JA, Nehler MR, Harris KA, Fowkes FG. Inter-society consensus for the management of peripheral arterial disease (TASC II). J Vasc Surg. 2007;45(Suppl S):S5-67. https://doi.org/10.1016/j.jvs.2006.12.037.

26. Chen DC, Singh GD, Armstrong EJ, Waldo SW, Laird JR, Amsterdam EA. Long-term comparative outcomes of patients with peripheral artery disease with and without concomitant coronary artery disease. Am J Cardiol. 2017;119(8):1146-52. https://doi.org/10.1016/j.amjcard.2016.12.023.
27. Heikkila K, Loftus IM, Mitchell DC, Johal AS, Waton S, Cromwell DA. Population-based study of mortality and major amputation following lower limb revascularization. Br J Surg. 2018;105(9):1145-54. https://doi.org/10.1002/bjs.10823.

28. Sigvant B, Lundin F, Wahlberg E. The risk of disease progression in peripheral arterial disease is higher than expected: a metaanalysis of mortality and disease progression in peripheral arterial disease. Eur J Vasc Endovasc Surg. 2016;51(3):395-403. https:// doi.org/10.1016/j.ejvs.2015.10.022.

29. Secemsky EA, Kundi H, Weinberg I, Schermerhorn M, Beckman JA, Parikh SA, et al. Drug-eluting stent implantation and longterm survival following peripheral artery revascularization. J Am Coll Cardiol. 2019;73(20):2636-8. https://doi.org/10.1016/j.jacc. 2019.02.020.

30. McDermott MM, Guralnik JM, Ferrucci L, Tian L, Kibbe MR, Greenland P, et al. Community walking speed, sedentary or lying down time, and mortality in peripheral artery disease. Vasc Med. 2016;21(2):120-9. https://doi.org/10.1177/1358863x15626521.

31. Rantner B, Kollerits B, Pohlhammer J, Stadler M, Lamina C, Peric $\mathrm{S}$, et al. The fate of patients with intermittent claudication in the 21 st century revisited-results from the CAVASIC study. Sci Rep. 2017;8:45833. https://doi.org/10.1038/srep45833.

Publisher's Note Springer Nature remains neutral with regard to jurisdictional claims in published maps and institutional affiliations. 